- A description of a patient presenting with $v C J D$ to a general dental practitioner.

- vCJD can present with atypical facial symptons such as paraesthesia.

- The case highlights the importance of medical history taking at each visit and the prompt referral of any patients with atypical signs and symptoms.

\title{
Presentation of a case of variant CJD in general dental practice
}

\author{
A. J. Smith ${ }^{1}$, D. I. Russell ${ }^{2}$, J. Greene ${ }^{3}$, A. Lowman ${ }^{4}$ and J. W. Ironside ${ }^{5}$
}

This case report describes the initial presentation of variant CJD to a general dental practitioner. The case highlights the importance of prompt referral of patients presenting with a history of atypical facial symptoms.

\section{CASE REPORT}

A 28-year-old patient presented to their general dental practitioner with a 4-week history of paraesthesia of the left side of the face including the lower lip. The patient was referred for an oral surgery consultant clinic appointment for a more detailed appraisal of the patient's signs and symptoms.

Examination at the consultant clinic revealed an unremarkable past dental and medical history. The patient complained of a 'pins and needles' sensation in the left cheek, left lower lip and chin and the skin

${ }^{1}$ Senior Lecturer, Microbiology, Glasgow Dental Hospital \&t School; ${ }^{2}$ Consultant Oral \& Maxillofacial Surgeon, Glasgow Dental Hospital \& School; ${ }^{3}$ Consultant Neurologist Institute of Neurological Sciences, Southern General Hospital, Glasgow; ${ }^{4}$ Registrar in Neurology, National CJD Surveillance Unit, University of Edinburgh, Western General Hospital, Edinburgh; ${ }^{5}$ Professor of Clinical Neuropathology, National CJD Surveillance Unit, University of Edinburgh, Western General Hospital, Edinburgh Correspondence to: A. J. Smith, Infection Research Group, Glasgow Dental Hospital \&t School, 378 Sauchiehall Street Glasgow G2 3JZ, Scotland

Email:a.smith@dental.gla.ac.uk

\section{Refereed Paper}

doi:10.1038/sj.bdj.4811468

Received 13.10.03; Accepted 16.01.04

๑ British Dental Journal 2004; 197: 75-76 of the left hand. Extra-oral examination demonstrated a partial loss of sensation as demonstrated by an impaired response to pin prick and light touch, as well as two point discrimination. This was evident in the areas of the left face supplied by the infra-orbital and mental nerves and the palm and back of the hand (C6, C7, C8). There was no weakness of the facial muscles and the right side was unaffected. Intra-oral examination revealed a healthy intact dentition with no obvious signs of dental disease. Due to the unusual distribution of the sensory abnormalities the patient was referred for an urgent neurological examination.

On presentation to the neurology clinic for a detailed neurological examination, 3 months after initial presentation to the dental practitioner, some change in the patients personality were noted. These included becoming increasingly withdrawn with episodes of confusion. Clinical examination revealed that the patient's sensory impairment on the left side had extended to include the left arm, leg and trunk. No other neurological signs and symptoms were observed. Approximately 1 month later further diagnostic tests were performed as follows:
- EEG - mild diffuse slowing

- MRI brain scan - bilateral diffuse areas of signal hypersensitivity affecting the pulvinar region of the brain

- CSF - 14-3-3 protein negative

- CSF - white cell count normal

On the basis of the clinical presentation of the sensory symptoms, psychiatric features and the MRI results a diagnosis of probable variant CJD was made. Eight months after presentation to the dental practitioner the patient died and the clinical diagnosis of variant CJD was confirmed following autopsy.

\section{DISCUSSION}

Psychiatric symptoms are common in the early stages of vCJD. Typical presentations are depression, anxiety and behavioural change. ${ }^{1}$ Neurological symptoms such as pain, paraesthesia and numbness precede psychiatric symptoms in 15\% of cases and are present in combination with psychiatric symptoms in $22 \%$ of cases from the onset of disease. The most common early neurological ( $<4$ months onset) features are persistent pain affecting the limbs, trunk and face but not associated with sensory symptoms. Within 4-6 months of onset paraes- 
thesia and numbness in a similar distribution to, and often associated with pain is common. ${ }^{2,3}$ A diagnosis of CJD has been confirmed following autopsy in all cases where a post-mortem examination has been performed. ${ }^{4}$

\section{DIAGNOSIS OF VCJD}

An ante-mortem diagnosis of vCJD can be difficult in the early stages of the disease. However, diagnosis can be facilitated by use of a number of diagnostic criteria based on a detailed clinical history of the nature of the symptoms, pattern of progression and duration of the illness and diagnostic tests, and can lead to a clinical diagnosis with a high degree of accuracy. ${ }^{4} \mathrm{~A}$ number of tests and investigations can contribute to a diagnosis:

\section{Blood tests}

In addition to excluding other forms of illness, blood tests collected with informed consent can be performed for any of the genetic mutations already identifiable in inherited prion diseases. Blood can also be tested for genetic susceptibility to vCJD by detecting the presence of methionine/ methionine homozygosity at codon 129 . To date, all cases of vCJD are homozygous for methionine at this location.

\section{EEG}

This can be a useful test for the diagnosis of sporadic CJD. In other forms of CJD the EEG may be normal or there may be nonspecific abnormalities.

\section{Cranial MRI}

An MRI scan can be useful in assisting a diagnosis. In vCJD, abnormal changes can be observed in the posterior thalamic area of the brain (pulvinar sign) and in SCJD there are occasional changes in the basal ganglia. $^{5}$

\section{CSF}

Analysis of CSF is useful to exclude some types of infection. An increase in the neuronal protein 14-3-3 in the CSF can be of considerable help in the diagnosis of sCJD, but it is less helpful in vCJD. ${ }^{6}$

\section{BIOPSY}

Brain biopsy is not recommended for any form of CJD unless an alternative treatable condition is suspected in the differential diagnosis. In vCJD, $\mathrm{PrP}^{\mathrm{Sc}}$ also accumulates outside of the CNS in lymphoid tissues, so that biopsy of tonsillar tissue can provide a means of a 'probable' diagnosis of vCJD. Within the UK, the use of tonsil biopsy for diagnosis of VCJD has been developed and validated. ${ }^{7}$

\section{CONCLUSION}

Although sensory disturbances in one or more divisions of the fifth cranial nerve is a rare presentation for VCJD, this possibility should be considered, particularly if the sensory symptoms progress to other areas. Some of the early clinical features of vCJD such as personality change, loss of concentration or sensory symptoms may occur in psychiatric conditions (sometimes as side effects of psychotropic drugs), or in the early stages of multiple sclerosis, However, the persistence of these symptoms over a number of weeks with no apparent cause should alert the practitioner to the presence of a more serious underlying cause and urgent referral for further investigation should be instituted. In particular, the emergence of ataxia (unsteady gait), myoclonus (sudden muscle spasms) or other movement disorders should strongly alert the clinician to suspect a progressive neurodegenerative condition such as vCJD.

The authors wish to acknowledge the kind support of the patient's family to allow us the opportunity to publish this report.

1. Spencer M D, Knight R S G, Will R G. First hundred cases of variant CJD: retrospective case note review of early psychiatric and neurological features. BrMed J 2002: 324: 1479-1482.

2. Henry C, Knight R. Clinical features of variant Creutzfeldt-Jakob disease. Rev Med Virol 2002; 12: 143-150.

3. Macleod M A, Knight R, Stewart G, Zeidler M, Will R. Sensory features of variant Creutzfeldt-Jakob disease. J Neurol Neurosurg Psychiatry 2000; 69: 413-414.

4. Will R G, Zeidler M, Stewart G E et al. Diagnosis of new variant Creutzfeldt-Jakob disease. Annals of Neurology 2000; 47: 575-582.

5. Zeidler M, Sellar R J, Collier D A et al. The pulvinar sign on magnetic resonance imaging in variant

Creutzfeldt-Jakob disease. Lancet 2000; 355: 1412 1418.

6. Hsich G, Kennedy K, Gibbs C J, Lee K H, Harrington M G. The 14-3-3 brain protein in cerebrospinal fluid as a marker for transmissible spongiform

encephalopathies. N Eng/J Med 1996; 335: 924-930.

7. Hill A F, Butterworth R J, Joiner S et al. Investigation of variant Creutzfeldt-Jakob disease and other human prion diseases with tonsil biopsy samples. Lancet 1999; 353: 183-189. 